\title{
Circuit
}

Musiques contemporaines

\section{Visages de Pousseur}

\section{Entrevue-concert avec Henri Pousseur, animée par Michel}

Gonneville, le 23 janvier 2001

\section{Portraits of Pousseur}

\section{Interview}

\section{Michel Gonneville}

Volume 12, numéro 1, 2001

Henri Pousseur : visages

URI : https://id.erudit.org/iderudit/902237ar

DOI : https://doi.org/10.7202/902237ar

Aller au sommaire du numéro

Éditeur(s)

Les Presses de l'Université de Montréal

ISSN

1183-1693 (imprimé)

1488-9692 (numérique)

Découvrir la revue

Citer ce document

Gonneville, M. (2001). Visages de Pousseur : entrevue-concert avec Henri Pousseur, animée par Michel Gonneville, le 23 janvier 2001. Circuit, 12(1), 19-28. https://doi.org/10.7202/902237ar
Résumé de l'article

Retranscription de l'entrevue réalisée par Michel Gonneville, inaugurant l'événement "Visages d'Henri Pousseur ». L'entrevue est concentrée sur deux moments clés de la carrière de Pousseur : son intégration au mouvement avant-gardiste des années 50, et son « refus du refus » au tournant des années 60. d'utilisation que vous pouvez consulter en ligne.

https://apropos.erudit.org/fr/usagers/politique-dutilisation/ 


\title{
Visages de Pousseur
}

\author{
Entrevue-concert avec Henri Pousseur, \\ animée par Michel Gonneville, \\ le 23 janvier 2001.
}

Michel Gonneville. - Comment un jeune homme belge né en 1929 en arrivet-il, vingt ans plus tard, à faire partie de l'avant-garde musicale internationale, des compagnons d'armes comme Boulez, Stockhausen, Berio?

Henri Pousseur. - D'entrée de jeu, il faut dire que je suis un petit campagnard. Je suis né dans une petite ville du fond des Ardennes belges, près de la frontière allemande, une ville francophone entourée de villages germanophones belges qui ont été réannexés à l'Allemagne pendant la Deuxième Guerre mondiale puisqu'ils l'avaient été avant la Première Guerre pour des raisons politiques très compliquées, liées aux guerres napoléonniennes. Cet épisode douloureux qu'a été la guerre avec cette réannexion a eu malgré tout, pour moi, un aspect positif, puisque j'ai eu au lycée un professeur de musique allemand qui était excellent. II a aussi été mon professeur de piano et il nous faisait chanter en chœur de la très bonne musique de la Renaissance ainsi que des fragments des Saisons de Haydn, par exemple. Cela a été un point de départ.

Après la guerre, quand nous avons été libérés, j'avais quinze ans... J'ai été, pendant quelques années, alors que je terminais mon lycée, dans une école de musique de la région. À dix-huit ans, en 1947, je me suis rendu au Conservatoire de Liège. J'avais envie depuis toujours de devenir musicien. "Depuis toujours", c'est peut-être beaucoup dire : j'aurais pu bifurquer vers d'autres disciplines, j'avais d'autres choix quand j'étais très jeune, mais j'ai assez vite décidé de me consacrer à la musique. À dix-huit ans, donc, je suis allé à Liège pour étudier le piano, l'orgue (i'étais très impressionné par cet instrument tout-puissant) et l'écriture (je voulais être compositeur). Je suis donc arrivé dans une vieille école poussiéreuse. Étant donné la situation politique dont j'ai parlé lle fait que nous avions été rattachés à l'Allemagnel et le fait que dans l'école de musique belge que j'avais fréquentée, il n'y avait pas de cours de solfège (ce n'était pas encore une école très réglementée, je suivais un cours de piano, d'orgue et d'harmonie), je n'avais pas fait de solfège au sens français du terme. J'avais appris à lire la musique, à l'entendre très bien, mais je ne possédais pas la technique de lecture des notes en 
les chantant. J'ai donc dû aller au cours de solfège qui a été pénible et que je considère comme très antimusical! Lors des dictées, cependant, tout le monde venait s'asseoir autour de moi pour copier, parce que j'entendais bien!

Lors des examens d'admission, le directeur m'avait dit : "Monsieur Pousseur, comme vous allez être à la classe d'orgue, il faudra que vous alliez au cours d'harmonie pratique et c'est monsieur Pierre Froidebise qui le donne. " Je suis donc allé dans la classe de Pierre Froidebise, qui était un jeune organiste et compositeur, très intéressé par la musique contemporaine, mais aussi par toute la musique du Moyen Âge à nos jours et par les arts extra-européens, japonais, chinois, par la philosophie, par des tas de choses! II avait des amis dans toutes les disciplines de la ville et il faisait partie d'une association qui diffusait la culture d'avantgarde. Peu de semaines après mon arrivée, il présentait une conférenceconcert qui reprenait un peu la démonstration de leibowitz (on venait de publier le premier livre de celui-ci, Schönberg et son école), démonstration selon laquelle I'histoire de la polyphonie occidentale est une progression linéaire continue qui va de tel compositeur à tel autre par la complexification harmonique croissante, l'ajout de nouvelles dissonances, etc., jusqu'à la dodécaphonie qui en est la conséquence logique. Froidebise avait repris cette démonstration d'une façon un peu moins rigide et certains de ses grands élèves, un peu plus âgés que moi, ont joué quelques pièces, l'un d'eux a même donné la création belge des Variations pour piano opus $27 \mathrm{~d}$ 'Anton Webern. J'ai été complètement sidéré par ce premier contact, très séduit par la démonstration analytique, explicative... Mes oreilles sont cependant restées un peu abasourdies. Cela m'a bouleversé, je suis sorti de là complètement secoué!

Michel Gonneville. - C'était en 1947, deux ans après la mort de Webern?

Henri Pousseur. - Oui et je me suis mis tout de suite à essayer de faire des séries, de les manipuler, etc. II m'a fallu deux ans pour que mes oreilles, qui étaient quand même déjà très prédéterminées de manière tonale et aussi un peu modale, finissent par accepter ces harmonies.

On avait toutes sortes d'activités. Moi, i'avais formé un petit chœur avec des universitaires. J'ai fait surtout chanter de la musique de la Renaissance et même un peu du Moyen Âge. On n'osait pas aborder le répertoire contemporain : c'était trop complexe. Mais on a eu, entre autres, la visite de Leibowitz à qui j'ai montré une première sonatine dodécaphonique que j'avais écrite deux ans plus tôt. Celuici m'a alors cité cette phrase de Schönberg : "On peut encore écrire beaucoup de bonne musique en do majeur ! » (Rires.) Le dialogue devait se terminer là !

Mes amis (des Liégeois et des Bruxellois) sont ensuite allés en groupe, une première fois, à Paris, rendre visite à de jeunes compositeurs, dont Boulez, et ils m'ont rapporté les dernières nouvelles. J'ai lu les articles de Boulez évidemment.

En 1951, j'avais 22 ans, Froidebise m'a amené avec lui à une quinzaine musicale qui avait lieu à Royaumont et où il y avait aussi, entre autres, Boris de 
Schloezer, Pierre Souvtchinsky, Max Deutsch et Pierre Boulez. On a passé quinze jours ensemble et ça a été pour moi quelque chose de tout à fait fantastique. Boulez n'a que quatre ans de plus que moi, mais il était déjà très avancé dans sa recherche et il avait déjà une certaine autorité. On a beaucoup parlé, notamment de Stockhausen dont il avait fait la connaissance. Par la suite, i'ai pris moi-même contact avec notre collègue allemand.

Les autorités du Conservatoire de Liège étaient très mécontentes de notre intérêt dominant pour la musique contemporaine et de tout ce que nous faisions. Nous avons donc eu des conflits et $j$ 'ai fini par claquer la porte pour aller terminer mes études à Bruxelles pendant mon service militaire. Déjà alors, une de mes œuvres avait été jouée au festival de la SIMC' à Salzbourg, en 1952, Trois Chants sacrés, que j'avais écrite peu de temps après avoir rencontré Boulez.

Michel Gonneville. - On est donc tout à fait plongé dans les années 50 avec leur période d'expérimentations, de recherches, de tabula rasa. Les justifications que vous vous donniez à ce moment-là de procéder à une telle recherche radicale, à un tel renouvellement du langage, quelles étaientelles?

Henri Pousseur. - C'est assez complexe, et on serait obligé d'explorer beaucoup de choses, mais pour rendre l'explication plus simple, j'aborderai la question de mon point de vue. J'étais le plus jeune du groupe, donc un peu influencé par les idées de mes aînés. Cependant, j'ai essayé très vite d'avoir ma réflexion personnelle comportant son aspect critique. Dans la démarche générale, il y avait une volonté de faire table rase. Pourquoi? Parce que nous sortions de la guerre et que nous avions l'impression (et nous n'étions pas les seuls, pas seulement les musiciens) que la culture occidentale officielle, la musique moderne néo-classique par exemple, tout ce qui essayait de conserver les valeurs du cartésianisme, d'un humanisme frileux et hypertrophié à la fois, c'est-à-dire d'une conception des choses mettant la conscience individuelle au centre du monde lavec toutes les conséquences qu'on sait sur le plan économique comme sur les plans philosophique, scientifique, etc.), nous avions l'impression que cette position-là était coupable, était en quelque sorte complice de ce qui avait pu arriver et qu'il fallait en sortir et commencer quelque chose de nouveau. Nous étions très naifs, très jeunes certes, mais nous avions cette forte sensation-là! Je crois que c'est une des raisons, parfois inconscientes et profondes qui ont été, entre autres, à l'origine de ce mouvement.

$\|$ y avait aussi des raisons historiques : les compositeurs de la génération précédente, du premier demi-siècle (il y en a toute une série) ont été très critiqués. Boulez a écrit des articles terribles sur Stravinsky, Bartók, etc., que j'ai désapprouvés un jour, mais pas immédiatement. Notre position était claire : "I n'y a que les Viennois! " Eł parmi eux, même Berg et Schönberg restaient très liés au passé tandis que Webern commençait quelque chose de vraiment nouveau. Il y avait là une espèce de germe qui a éliminé au maximum les résidus et à partir duquel on pouvait construire quelque chose de frais, de régénéré.
1. Société internationale de musique contemporaine 
Michel Gonneville. - II y avait des raisons philosophiques, mais il y avait aussi des chocs musicaux...

Henri Pousseur. - Oui, naturellement, on ne peut nier l'aspect "virginal » de tout cela! II y avait aussi des idées constructivistes liées aux déclarations d'autres compositeurs comme Stravinsky, par exemple, qui disait que «la musique n'exprime pas", ce qui était naturellement une déclaration très partiale et partielle! II voulait dire qu'on n'exprimait plus tellement les sentiments romantiques. C'était en effet, selon moi, une réaction anti-romantique que nous partagions jusqu'à un certain point et certains de mes amis plus que d'autres.

La première musique de Boulez est une musique quand même très expressionniste... Mais il y a eu un moment où tout ça fut réprimé au profit du constructivisme...

Michel Gonneville. - Stimulé, entre autres, par la fameuse étude de Messiaen ${ }^{2}$...

Henri Pousseur. - Oui, l'influence de Messiaen a été très importante. Messiaen n'était pas du tout un fanatique du constructivisme exclusif, mais il avait fait ce travail de recherche.

Quand je l'ai rencontré, Boulez était en train d'écrire Polyphonie $X$, qu'il a retiré plus tard; il était encore en train d'achever le premier livre de Structures et il voulait intituler cette œuvre: $\dot{A}$ la limite du pays fertile selon le titre d'un tableau de Paul Klee qui s'appelle: Monument à la limite du pays fertile. II disait que c'était une musique où on essayait de se passer de tout notre héritage "pour voir ce qui reste " et où on mettait en œuvre des techniques de constructions ultracérébrales, quasi scientifiques ou mathématiques, pour y arriver; celles-ci amenaient des résultats qui n'étaient pas complètement étrangers aux techniques de hasard mises en œuvre par John Cage. D'ailleurs, nous avions beaucoup de contact avec l'école américaine.

Michel Gonneville. - On vient de parler des années où tu as constitué avec d'autres un langage différent. Quelques années plus tard tu vas écrire la Chevauchée fantastique, extraite de Votre Faust $3^{3}$. Parlons très brièvement de cette œuvre et nous pourrons peutêtre ensuite parler plus longuement de ce tournant des années 60 .

Henri Pousseur. - Oui, en 1960, j'ai décidé de quitter l'exclusivisme étroit de la musique sérielle, qu'on appelait alors "généralisée " et que plus tard j'ai appelée « restreinte". J'ai essayé de faire ensuite de la série beaucoup plus généralisée en l'étendant à toutes sortes d'autres domaines et, en particulier, en ne refusant plus la liaison au passé, notre héritage, nos racines; non pas pour faire du néoclassicisme, mais pour mieux répondre au présent et à l'avenir immédiat.

$\|$ y a donc eu un travail sur l'Histoire. J'ai écrit avec Michel Butor cette espèce d'opéra mobile qui s'appelle Votre Faust et qui est aussi une énorme architecture
2. Mode de valeurs et d'intensités, écrit à Darmstadt en 1949 et faisant partie des Quatre Études de rythme pour piano solo.

3. Lors de l'entrevue, La Chevauchée fantastique fut exécutée par un jeune pianiste du Conservatoire, Olivier Godin. 
de citations... Mais il y avait en même temps la recherche d'une grammaire générale qui justifie l' être-ensemble" de toutes ces citations, qui ne se contente pas simplement de les accoler, qui les intègre, les fait apparaître comme des cas spéciaux possibles, de cette supergrammaire.

Il y a dans cet opéra, dans la deuxième partie, qui est celle où le public peut intervenir pour choisir la manière dont l'histoire va évolver, une scène de foire en quatre versions différentes, dans laquelle il y a un petit spectacle de pseudomarionnettes, un petit Faust, très rapide (de cinq à dix minutes selon la version). C'est donc un théâtre sur le théâtre, où l'histoire du Faust "principal », qui est un jeune compositeur, se reflète en quelque sorte (il prend conscience comme dans un miroir de son histoire à luil.

Autour de ce spectacle de marionnettes, la musique de foire comporte beaucoup de citations d'opéra du XIX siècle / $c^{\prime}$ est une grande salade, en quelque sorte) et le spectacle de marionnettes lui-même comporte une espèce de fond musical, de scènes musicales enveloppantes. Pour deux des quatre versions, ce sont des scènes musicales de grande valeur, qui m'étaient très chères, que j'avais vues (au cinéma) et entendues, adolescent, et que j'aimais beaucoup... La scène du commandeur, par exemple, dans Don Giovanni de Mozart, et la scène des Enfers de l'Orphée de Gluck. Les deux autres versions, ce sont des pseudo grandes citations dont l'une est une fantaisie pour piano qui commence comme du Haydn et qui évolue en quatre ou cinq minutes, à travers tout le $x x^{\mathrm{e}}$ siècle jusqu'à Schönberg. Webern et moi-même, mais d'une façon extrêmement continue, sans qu'on sente les coutures. C'est un peu une satire de la démonstration de Leibowitz. En fait, La Chevauchée fantastique, c'est le titre d'une des estampes de Delacroix sur Faust, et Michel Butor l'a utilisé comme cri d'un montreur de spectacle dans les différentes situations du spectacle de marionnettes... Je l'ai repris comme titre de cette pièce-ci, à l'intérieur d'une œuvre satellite pour le concert, Miroir de Votre Faust, qui est composée de trois parties dont celle-ci est la partie centrale et la plus courte, les autres étant des mobiles...

Michel Gonneville. - Henri, nous voici rendus aux années où tu vas, comme tu dis, "refuser le refus". C'est un tournant important dans ta carrière de compositeur et qui est motivé par toutes sortes de raisons que j'aimerais que tu nous exposes. II ne s'agit pas de nostalgie... II y a peutêtre une perspective à partir de laquelle tu pourrais répondre à cette question lorsque tu dis et je te cite: "ce tournant accomplit mieux ma fidélité webernienne profonde "... Alors là, on peut se demander comment on passe de Webern à la Chevauchée fantastique, par exemple, et à d'autres œuvres d'Henri Pousseur comme Vue sur les jardins interdits.

Henri Pousseur. - Les deux ont une propriété commune, c'est de réaliser, d'une manière continue, par une progression régulière, la compatibilité de langages qui paraissaient jusqu'alors incompatibles. II faudrait peutêtre que je donne l'interprétation que j'ai essayé de dégager de l'esthétique de Webern, car très tôt je me 
suis posé cette question: "Pourquoi est-ce que l'œuvre de Webern nous fascine tellement et pourquoi est-ce que nous éprouvons le besoin d'en faire notre point de départ et le phare de notre propre recherche? »

Webern a travaillé toute sa vie à chercher quelque chose qu'il a d'ailleurs trouvé très tôt, même avant la période dite dodécaphonique au sens strict. Vous savez comme moi que Schönberg, avec l'aide de ses grands disciples, a mis au point, au début des années 20 , après un long silence et une grande période de musique dite atonale, le système dodécaphonique au sens strict, c'est-à-dire celui des séries de douze sons qui sont utilisées d'une certaine manière, très réglementée. Or, pendant la période atonale, Webern avait déjà trouvé son esthétique comme en témoigne toute une série d'œuvres: les Bagatelles pour quatuor opus 9 et la série de lieder jusqu'à l'opus 17. Les œuvres du début des années 20 renferment déjà les premiers rudiments de dodécaphonie et confirment une mise en place de l'esthétique webernienne au point de vue de ce que je pourrais appeler l'harmonie. II ne s'agit plus du tout d'une harmonie au sens classique, ce n'est plus une harmonie d'accords enchaînés, comme on peut encore la trouver chez Schönberg à la rigueur et chez Berg en particulier, mais une harmonie de notes isolées qui forment ce que j'appelle des constellations. Ce qui est caractéristique chez Webern, c'est qu'on entend des constellations de corps lumineux... Quand j'ai entendu cela pour la première fois, et surtout à mesure que je l'entendais, j'ai été absolument frappé ! La manière dont ces notes se situaient dans l'espace, sans aucune pesanteur, me fascinait! Stravinsky lui-même, dix ans après la mort de Webern, en 1955, quand nous étions déjà en plein travail autonome, dont il a eu d'ailleurs connaissance, a écrit un petit texte pour la revue que publiait Stockhausen et à laquelle je participais, un petit texte dans lequel il a écrit : "Webern est le héros qui a taillé ses diamants dans la solitude. "Vous voyez! Moi, je parlais d'étoiles dans le ciel et lui parlait de diamants qui brillent dans la nuit... C'est un peu la même image. Eł Webern lui-même a dit à la fin de sa vie: "J'ai enfin trouvé un nouveau type de tonalité où les notes ne se rapportent plus que les unes aux autres», selon la prescription de Schönberg, c'est-à-dire qu'il n'y a plus de notes dominantes, plus aucune note qui attire les autres à soi ; elles ont toutes la même valeur ou le même poids, bien qu'elles aient toutes une couleur et une personnalité différentes. Chacune est à sa place et elles se maintiennent en équilibre. Tout reste suspendu. C'est vraiment l'inverse de la tonalité, ce n'est plus du tout le sujet qui est le centre. Dans la musique tonale, tout se rapporte à une note que vous entendez comme votre point d'équilibre personnel. C'est l'ego qui est symbolisé par la tonique et tout s'organise autour de votre conscience...

Michel Gonneville. - C'est comme un point de gravité...

Henri Pousseur. - Oui! Alors que dans le système webernien, au contraire, c'est un microcosme par rapport auquel on se situe soi-même comme un des points. Cela suppose donc une nouvelle compréhension du rapport de l'individu à l'univers, à la société, à ses autres partenaires, une égalité dans la différence. 
Michel Gonneville. - Et alors, le tournant?

Henri Pousseur. - Oui, le tournant... (Rire.) C'est que nous avons, dans les années 50, vécu en essayant de généraliser l'exemple de Webern, en multipliant, en épaississant, en allongeant, en faisant une soupe beaucoup plus consistante à partir du même vocabulaire. On a essayé, par différentes manières, de le dépasser, en allant, par exemple, plus vers le bruit avec la musique électroacoustique ou en faisant des œuvres ouvertes, ce qui était une conséquence de cette situation puisqu'il n'y avait plus de notes centrales. On pouvait donc imaginer que ça pouvait se tourner de différentes manières. Dans les constellations weberniennes d'ailleurs, on a des exemples (le début du Concerto opus 24, par exemple) où une même constellation se tourne de plusieurs manières, comme un cristal que l'on regarde de différents côtés. C'est une espèce de mobilité.

Personnellement, je me suis dit qu'il fallait continuer à ouvrir le champ et qu'on avait besoin de reprendre tous les éléments qu'on avait exclus, car on en avait besoin pour construire quelque chose de beaucoup plus vaste, de beaucoup plus général, mais à la condition toutefois que le principe webernien reste valable, c'està-dire qu'on fasse des constellations multipolaires ou omnipolaires, non plus de note à note nécessairement, mais de bloc de musique à bloc de musique, de style à style, de type d'harmonie à type d'harmonie, etc. On peut donc avoir, par exemple, une harmonie renaissante jouant avec une harmonie romantique, mais on peut aussi avoir une harmonie tonale jouant avec une harmonie non tonale ou avec des bruitages, à la condition qu'on arrive à mettre tout cela en harmonie, sur le même dénominateur. II faut donc, pour cela, développer une pensée théorique très complexe qui soit, en quelque sorte, métagrammaticale...

Michel Gonneville. - Eł non seulement une pensée théorique, mais aussi des techniques...

Henri Pousseur. - des techniques de construction, oui...

Michel Gonneville. - L'une d'entre elles est la technique des réseaux qui permet justement d'établir une continuité entre une harmonie dissonante et une harmonie plus consonante, par exemple. Et c'est un peu une technique apparentée à celle des réseaux que tu as utilisée dans Vue sur les jardins interdits.

Henri Pousseur. - Je n'ai pas inventé cette technique des réseaux. C'est une technique que i'ai progressivement découverte en me posant le problème suivant : "Comment vais-je faire? " Je me suis dit: "Je vais maintenant écrire une œuvre de grande dimension où je vais essayer de réaliser ce projet», qui a été Votre Faust. Je me suis mis à l'école de l'Histoire, non seulement en faisant du collage mais en développant une grammaire de telle sorte que chaque style historique remplisse une case parmi d'autres, avec des cases qui n'étaient pas encore occupées, qui se développent entre celles qu'on connaît et même au-delà ly compris le bruit ou le son complexel. J'ai trouvé que dans toute l'histoire de la théorie musicale, de la 
grammaire harmonique, il y avait quelque chose de commun. La musique de Webern est constituée d'espèces de réseaux, c'està-dire de structures économiques où il y a un choix d'intervalles bien précis qui régit toute la distribution des sons. Le chromatisme, d'abord, est très important, mais c'est surtout un chromatisme de grande distance, c'est-à-dire des septièmes majeures, des neuvièmes mineures, donc des intervalles qui annulent l'octave (l'octave étant un intervalle qui identifie des notes de différents registres les unes par rapport aux autres). II y a donc, par exemple, une note qui va se placer "à côté", qui empêche la perception octaviante, et qui fait que l'espace devient différent, courbe, "non euclidien". $\|$ y a d'autres intervalles qui viennent s'y joindre, mais qui forment des losanges, des parallélogrammes (deux notes à distance de septième majeure et des tierces mineures dans l'autre sens, par exemple, forment des constellations). Parfois c'est un peu plus complexe. II y a plusieurs manières d'aborder ce concept, mais tout peut se réduire à des réseaux. De même, la modalité ancienne, monodique, peut se résumer à un réseau d'octaves et de quintes. Si on ajoute la tierce majeure, on obtient le réseau de la tonalité, qui n'est plus à deux, lui, mais à trois dimensions, un peu comme la perspective italienne ou l'espace cartésien avec ses trois coordonnées. On peut déformer ces réseaux en remplaçant l'octave, non seulement par une septième majeure, comme chez Webern, mais par une neuvième ou une dixième, comme chez Stravinsky, par exemple. On peut faire aussi toute sorte de combinaisons, connues ou inconnues, y compris avec des micro-intervalles /on obtient alors des musiques non européennes). Et tout d'un coup, on a un outil d'analyse et de composition qui permet de voyager à travers I'Histoire et la géographie... C'est prodigieux!

Michel Gonneville. - Justement, dans la perspective géométrique que tu évoquais précédemment, dans Vue sur les jardins interdits, on entend un travail de ce type sur les intervalles. On part d'intervalles assez dissonants et peu à peu on se rapproche d'un modèle qui est une espèce de danse ou de choral dansé de Samuel Scheidt, un organiste du XVII siècle...

Henri Pousseur. - En fait, j'avais déjà fait ça avec une pièce, un peu avant, Couleurs croisées...

Michel Gonneville. - C'est tout à fait différent de La Chevauchée fantastique, qui est une espèce de grand pastiche en évolution tandis qu'ici on se dirige vers un modèle qui ressort du contexte, un modèle connu, un style particulier, et puis qui redisparaît peu à peu...

Henri Pousseur. - Oui, il faut dire que dans la Chevauchée fantastique, il y a des citations de Schubert, de Schumann, de Liszt, de Wagner. Même le début, c'est Gluck, mais varié! Et puis la valse qui apparaît est basée sur un motif qui est une variation de l'air de Gluck. Ce n'est pas simplement du collage, car c'est très soigneusement cousu et travaillé. Alors que dans Vue sur les jardins interdits, il y a un seul modèle qui convenait particulièrement bien. Cette pièce commence 
avec des sons presque électroacoustiques et, petit à petit, l'œuvre s'articule de telle manière qu'on devine des espèces de motifs encore très simples, quasi weberniens qui deviennent des lignes, puis des petits tourbillons. Soudain, il y a quelque chose qui semble évoquer un rythme qui pourrait être celui d'une danse, puis il y a deux accords parfaits qui apparaissent brièvement, puis moins brièvement et puis il y a toute une petite phrase! Finalement, le choral est là pour un moment, puis il s'arrête, et brusquement on repart dans l'autre sens! la fin est bâtie sur des espèces de grands accords statiques qui retournent vers le son presque électronique du début, mais selon un chemin différent.

Pendant que j'écrivais cette dernière partie (je n'ai pas écrit du début à la fin, d'ailleurs. Je suis parti du choral et j'ai écrit ensuite dans les deux sens), un de mes vieux amis, compositeur et chef $d^{\prime}$ 'orchestre célèbre, Bruno Maderna, est mort. C'était une grande perte et j'ai été très affecté. On entend cela, je crois, dans la fin qui fait un peu penser, me semble-til, au choral dont Stravinsky a tiré les Symphonies d'instruments à vent et qu'il avait écrit pour souligner la mort de Claude Debussy. II y a quelque chose de commun dans cette espèce de statisme d'accords. Vue sur les jardins interdits est une pièce qui date de 1973. C'était une commande pour un quatuor de saxophones ${ }^{4}$. J'en ai fait d'autres versions, avec exactement les mêmes notes, mais pour d'autres effectifs.
4. Vue sur les jardins interdits fut exécutée lors de l'entrevue par un quatuor de saxophones formé d'élèves avancés du Conservatoire. 


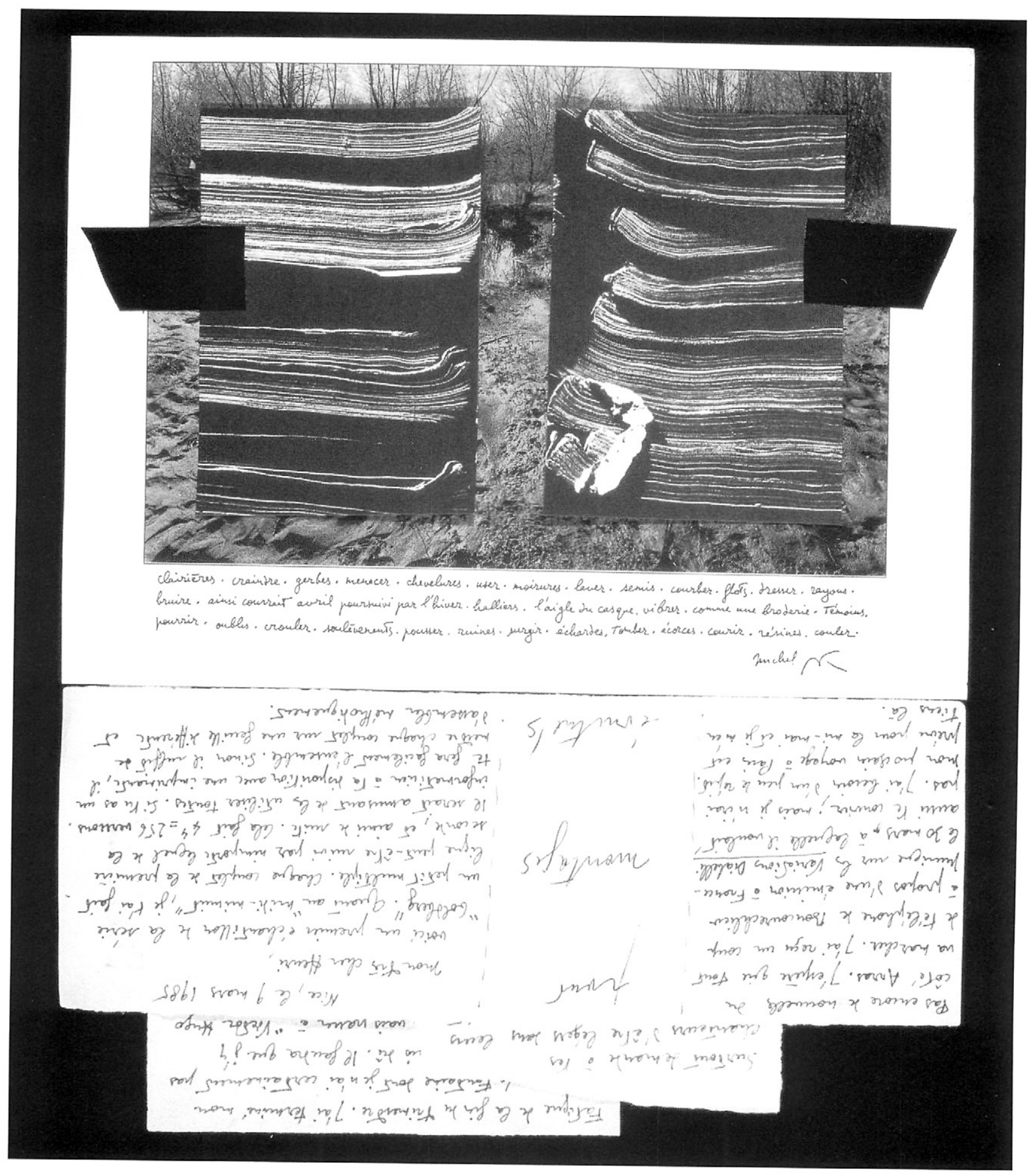

